\title{
Long Term Care and Vitamin D Supplementation in COVID-19 ERA
}

\author{
Carmine Finelli ${ }^{1,2 *}$ \\ ${ }^{1}$ Department of Internal Medicine, Ospedale Cav. R. Apicella- ASL Napoli 3 Sud, Via di Massa, 1, 80040 Pollena (Napoli), Italy \\ ${ }^{2}$ Covid Hospital Boscotrecase - ASL Napoli 3 Sud, Via Lenza, 3, 80042 Boscotrecase (Napoli), Italy
}

*Corresponding author: Carmine Finelli, Department of Internal Medicine, Ospedale Cav. R. Apicella-ASL Napoli 3 Sud, Via di

Massa, 1, 80040 Pollena (Napoli), Italy

\begin{tabular}{|c|c|}
\hline ARTICLE INFO & ABSTRACT \\
\hline Received: 慧April 15, 2021 & $\begin{array}{l}\text { Citation: Carmine Finelli. Long Term Care and Vitamin D Supplementation in COVID-19 } \\
\text { ERA. Biomed I Sci \& Tech Res 35(3)-2021. BISTR. MS.ID.005699. }\end{array}$ \\
\hline
\end{tabular}

\section{Editorial}

Coronavirus disease 2019 (COVID-19) outbreaks have been recorded in long-term care facilities around the world, ranging from nursing homes to rehabilitation clinics [1]. Long-term care patients (or residents) need continuous care, live in close quarters, and are typically elderly and multimorbid, placing them at a higher risk of contracting SARS-CoV-2 (the virus) and suffering severe COVID-19 consequences [2,3]. Vitamin D deficiency has been linked to SARS-CoV-2 sensitivity and disease progression [4]. Educational services, nutritionists, and other doctors may be able to extend this information to social media health platforms and forums used by adults for specific vitamin D-containing nutrients, ensuring that what people have been exposed to is based on science and provides for particularly feasible, safe, and low-cost solutions and activities, especially during the winter months [5]. Long-term care facility patients are prone to vitamin D deficiency. The endogenous synthesis of vitamin D requires skin exposure to the sun, which is in short supply for most residents in long-term care facilities.

Although vitamin D is present in small amounts in some foods, it is difficult to prevent vitamin D deficiency without dietary supplementation in the absence of sufficient sun exposure. Osteomalacia and osteoporosis are also caused by a lack of vitamin D [6]. It may also lead to muscle fatigue and a higher risk of falling [7]. Because the symptoms of vitamin D deficiency are so vague, either screening or regular supplementation is necessary. There isn't enough evidence to recommend that all nursing home residents be tested for vitamin D deficiency. The single best test

for determining whether a patient is vitamin D deficient is serum 25-hydroxyvitamin D. Despite the relative cost of the test and the high prevalence of vitamin D deficiency in this population, regular vitamin D supplementation should be suggested for all nursing home residents [8]. During the examination of residents newly diagnosed with osteoporosis, a serum 25-hydroxyvitamin D level, parathyroid hormone level, and calcium level should all be taken into account [8].

These tests aren't needed before starting vitamin D supplementation on a regular basis. Vitamin D supplementation is not recommended for people who have hypercalcemia or have signs of vitamin D toxicity. Supplementing with vitamin D is not recommended for people who are hypersensitive to any ingredient of a vitamin D-containing product. By inhibiting vitamin D 25-hydroxylase activity in the liver, phenobarbital and phenytoin can lower plasma levels of 25-hydroxyvitamin D. It's unclear what kind of treatment will be best in this situation. The elderly and chronically ill patients, who are at high risk for vitamin D deficiency, have a higher disease incidence and mortality rate due to COVID-19 infection. Inflammation and immune function are also supported by vitamin D [9]. Vitamin D supplements are available in two forms: vitamin D2 (ergocalciferol) and vitamin D3 (cholecalciferol). Vitamin D comes in the form of cholecalciferol, which is found in nature. A standard daily dose is 200-400 IU (5-10 micrograms).

Vitamin D and calcium are often mixed in one tablet. The amount of vitamin $\mathrm{D}$ that should be given as a dietary supplement 
varies depending on the source. At a minimum, 400 IU should be delivered effectively, but for older nursing home residents, 800$1000 \mathrm{IU}$ is a better dosage. In older people, $400 \mathrm{IU}$ of Vitamin D per day has been shown to be inadequate to avoid fractures [10]. This is a worthwhile measure, given the high mortality and disability correlated with hip fractures in this population, as well as the low cost and side effects of supplementation. Vitamin D is not present in all multivitamins in sufficient quantities. When measuring the dosage of supplementary vitamin $\mathrm{D}$, the quantity of vitamin $\mathrm{D}$ in a multivitamin should be taken into account. Nephrocalcinosis can be caused by consuming too much vitamin D. Because of irreconcilable constipation injury induced by the calcium, some long-term care residents obtaining a mixture of calcium and vitamin D may not be able to handle this procedure. In conclusion, for older patients in long term care, a daily calcium intake of $1200 \mathrm{mg}$ is suggested, and vitamin D supplementation should be continued if calcium supplementation is to be stopped.

\section{Disclosure Statement}

The author declare that there are no conflicts of interest.

\section{References}

1. Finelli C (2021) Long Term Care, Frailty and SARS-COV-2 Infection: A Framework of Situation. EC Clinical and Medical Case Reports 4: 47-48.

\section{ISSN: 2574-1241}

DOI: $10.26717 /$ BJSTR.2021.35.005699

Carmine Finelli. Biomed J Sci \& Tech Res

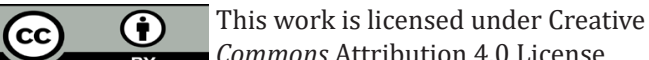

Submission Link: https://biomedres.us/submit-manuscript.php
2. Finelli C (2021) Obesity and the Frailty Syndrome at Period of Covid-19. Biomed J Sci \& Tech Res 33(5).

3. Finelli C (2021) Long-term Care, Malnutrition and Covid-19: A Framework of Situation. Journal of Medicine and Healthcare 3(1): 1-2.

4. Pizzini A, Aichner M, Sahanic S (2020) Impact of Vitamin D Deficiency on COVID-19-A Prospective Analysis from the CovILD Registry. Nutrients 12(9): 2775.

5. Finelli C (2021) A Possible Correlation between Obesity, Covid-19 and Vitamin D. Interventions Obes Diabetes 4(5): IOD.000599.

6. Sitta Mdo C, Cassis SV, Horie NC, Moyses RM, Jorgetti V, et al. (2009) Osteomalacia and vitamin D deficiency in the elderly. Clinics (Sao Paulo) 64(2): 156-158.

7. Parijat P, Lockhart TE (2008) Effects of lower extremity muscle fatigue on the outcomes of slip-induced falls. Ergonomics 51(12): 1873-1884.

8. Hamid Z, Riggs A, Spencer T, Redman C, Bodenner D (2007) Vitamin $\mathrm{D}$ deficiency in residents of academic long-term care facilities despite having been prescribed vitamin D. J Am Med Dir Assoc 8(2): 71-75.

9. Bassatne A, Basbous M, Chakhtoura M, Zein OE, Rahme M, et al. (2021) The link between COVID-19 and VItamin D (VIVID): a systematic review and meta-analysis [published online ahead of print, 2021 Mar 24] Metabolism 119: 154753

10. Reid IR, Bolland MJ (2020) Calcium and/or Vitamin D Supplementation for the Prevention of Fragility Fractures: Who Needs It? Nutrients 12(4): 1011.

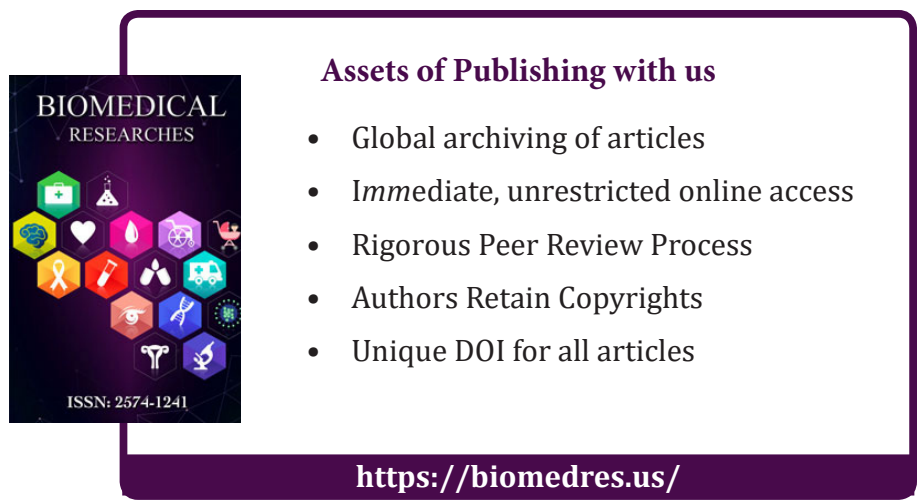

